\title{
TRANSPORT, GROWTH MECHANISMS, AND MATERIAL QUALITY IN GaN
}

\section{EPITAXIAL LATERAL OVERGROWTH}

\author{
Michael E. Coltrin, Christine C. Willan, Michael E. Bartram, Jung Han, \\ Nancy Missert, Mary H. Crawford, Albert G. Baca
}

Sandia National Laboratories, Albuquerque, NM 87185

Cite this article as: MRS Internet J. Nitride Semicond. Res. 4S1, G6.9(1999)

\begin{abstract}
Growth kinetics, mechanisms, and material quality in GaN epitaxial lateral over-growth (ELO) were examined using a single mask of systematically varied patterns. A 2-D gas phase reaction/diffusion model describes how transport of the Ga precursor to the growth surface enhances the lateral rate in the early stages of growth. In agreement with SEM studies of truncated growth runs, the model also predicts the dramatic decrease in the lateral rate that occurs as $\mathrm{GaN}$ over-growth reduces the exposed area of the mask. At the point of convergence, a step-flow coalescence mechanism is observed to fill in the area between lateral growth-fronts. This alternative growth mode in which a secondary growth of $\mathrm{GaN}$ is nucleated along a single convergence line, may be responsible for producing smooth films observed to have uniform cathodoluminescence (CL) when using $1 \mu \mathrm{m}$ nucleation zones. Although emission is comprised of both UV $(\sim 365 \mathrm{~nm})$ and yellow $(-550 \mathrm{~nm})$ components, the spectra suggest these films have reduced concentrations of threading dislocations normally associated with non-radiative recombination centers and defects known to accompany growth-front convergence lines.
\end{abstract}

\section{INTRODUCTION}

$\mathrm{GaN}$ grown on sapphire or $\mathrm{SiC}$ has a high defect density due to a significant lattice mismatch at the substrate-material interface. This high defect density $\left(>10^{9}\right.$ per $\left.\mathrm{cm}^{2}[1]\right)$ contributes to the poor electrical and optical materials quality [2-4]. There have been many demonstrations that epitaxial lateral overgrowth (ELO) can reduce the dislocation density in GaN films grown by metal-organic chemical vapor deposition (MOCVD) [5-7]. This reduction is the key to fabricating optoelectronic and electronic devices with high performance and reliability.

It has been shown that ELO feature morphology is influenced by several factors that include temperature, V/III ratio, and the mask fill factor [8-11]. The manipulation of these factors results in the cross-section morphology changing systematically from triangles, to fivesided polygons, to rectangles. The manner in which these initial growth features converge and coalescence into continuous films has a direct effect on optical quality and uniformity of the resulting smooth film.

\section{EXPERIMENT}

A $2 \mu \mathrm{m}$ thick GaN base layer was grown by MOCVD in a rotating disk reactor on 2-in diameter sapphire wafers using a low temperature buffer layer followed by high temperature planar growth. This was then covered with $1000 \AA \mathrm{SiO}_{2}$ or $\mathrm{Si}_{3} \mathrm{~N}_{4}$ and patterned using standard 


\section{DISCLAIMER}

This report was prepared as an account of work sponsored by an agency of the United States Government. Neither the United States Government nor any agency thereof, nor any of their employees, make any warranty, express or implied, or assumes any legal liability or responsibility for the accuracy, completeness, or usefulness of any information, apparatus, product, or process disclosed, or represents that its use would not infringe privately owned rights. Reference herein to any specific commercial product, process, or service by trade name, trademark, manufacturer, or otherwise does not necessarily constitute or imply its endorsement, recommendation, or favoring by the United States Government or any agency thereof. The views and opinions of authors expressed herein do not necessarily state or reflect those of the United States Government or any agency thereof. 


\section{DISCLAIMER}

Portions of this document may be illegible in electronic image products. Images are produced from the best available original document. 
photolithography techniques. Sub-sets of dot and line patterns, varied systematically with respect to both nucleation and mask dimensions were included in a single pattern. The dimensions in each sub-set are denoted as w:m, where $w$ is nucleation width (varied from $1 \mu \mathrm{m}$ to $8 \mu \mathrm{m}$ ) and $\mathrm{m}$ is the masked width (varied from $1 \mu \mathrm{m}$ to $32 \mu \mathrm{m}$ ). A fill factor $\theta$ is defined as $w /(w+m)$. Line and dot patterns were oriented both in the preferential ELO $<1-100>$ direction and in the $<1-$ $210>$ direction, which yields a smaller lateral to vertical growth ratio [5]. The ELO growth was performed at substrate temperatures of $1000^{\circ} \mathrm{C}, 1050^{\circ} \mathrm{C}$, or $1090^{\circ} \mathrm{C}$ using $\mathrm{H}_{2}$ as a carrier gas. The total pressure was 140 Torr with a TMG flow rate of $18 \mathrm{sccm}$ and $44 \% \mathrm{NH}_{3}$. An in-situ optical growth monitor was used to measure the growth rate of a center control section with a 1 $\mathrm{cm}$ diameter that contained no patterning. Samples were characterized by scanning electron microscopy (SEM) and cathodoluminescence (CL).

\section{MODEL OF ELO TRANSPORT LIMTTATIONS}

In ELO, growth does not occur on the dielectric masking material. Thus, the Ga precursor is not consumed (depleted) above the mask, creating an extra supply of reactants that can be transported via gas-phase diffusion to unmasked areas. This additional source of Ga species leads to growth rate enhancement on the unmasked regions, which will drop-off with distance from the mask. We have constructed a steady-state (time-independent) 2-D finite-difference model of the gas-phase diffusive transport and growth-rate enhancement of GaN during ELO.

The model solves the diffusion equation (Laplace's equation) for the Ga concentration, $\mathrm{n}$, in 2-D,

$$
\frac{\partial^{2} n}{\partial x^{2}}+\frac{\partial^{2} n}{\partial y^{2}}=0
$$

using a simple iteration scheme. The top boundary condition specifies that the concentration is a constant across the domain. Zero-flux boundary conditions are used at the left and right boundaries. At the surface, there is a zero-flux boundary condition on masked regions. For exposed regions, the boundary condition states that the diffusive flux of Ga species to the surface equals the rate of destruction due to deposition chemistry, i.e.,

$$
D \frac{\partial n}{\partial y}=k n,
$$

where $D$ is the diffusion constant (with units $0 \mathrm{~cm}^{2} / \mathrm{s}$ ), and $k$ is the surface reaction rate constant (with units of $\mathrm{cm} / \mathrm{s}$ ).

The model calculates the growth rate enhancement, i.e., the growth rate in the unmasked region divided by the normal growth rate when masking (ELO) is not used. The only parameter needed in the model is the ratio $D / k$. The diffusion constant is estimated from previous work modeling GaAs growth [12] to be $28.1 \mathrm{~cm}^{2} / \mathrm{s}$ at $1050^{\circ} \mathrm{C}$ and 140 Torr. A surface reaction probability of one for the Ga species corresponds to a rate constant $k$ of $24,700 \mathrm{~cm} / \mathrm{s}$ [13], which yields a value of $D / k$ of $11.4 \mu \mathrm{m}$. Using a unit reaction probability might seem unreasonable. However, since the system is transport limited, results of the model are insensitive to the value of the rate constant used. Figure 1 shows the calculated growth rate (normalized by the growth rate $g_{0}$ for the case of no masking) for patterns in the series $\mathrm{x}:(5-\mathrm{x}), \mathrm{x}:(9-\mathrm{x})$ and $\mathrm{x}:(18-\mathrm{x})$ plotted as a function of $\theta$.

A very large growth-rate enhancement is seen at small $\theta$, a factor of 6.6 for a 1:8 $(\theta=0.111)$ pattern. The growth rate slows dramatically as the ratio of the exposed to unexposed areas increases, dropping to 3.75 at $\theta=0.222$, for example. For the range of feature dimensions that we studied, the growth-rate enhancement depended only on $\theta$, and not on $\mathrm{w}$ and $\mathrm{m}$. For example, 
the calculated growth rate on a 1:8 feature matches that on a $2: 16$ feature to within $0.5 \%$. The calculated growth rate $g$ was empirically observed to have a power-law dependence on the fillfactor, i.e.,

$$
g=g_{o} \theta^{-0.862},
$$

where $g_{0}$ is the growth rate where no masking is used. The solid curve in Figure 1 is the fit from Eq. (3), which describes the 2-D transport results almost exactly.

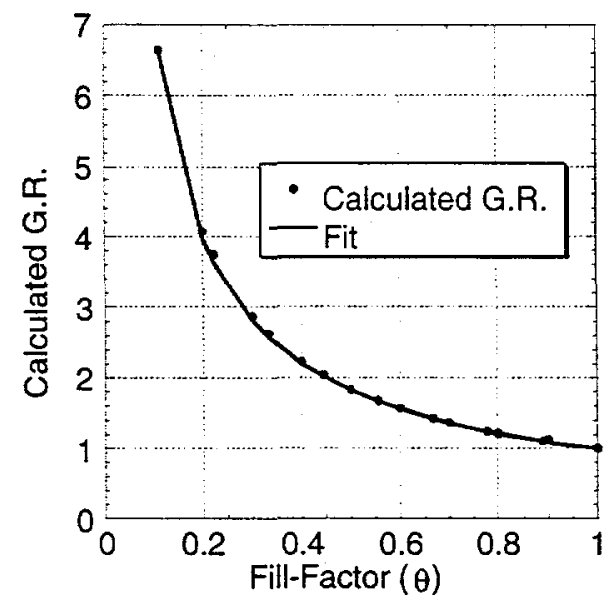

Figure 1. Growth rate $g$ (normalized by the unenhanced growth rate $g_{0}$ ) calculated by the 2-D transport model as a function of fill-factor $\theta$. Solid line shows the fit from Eq. (3).

One interesting feature of the model is that the total growth rate averaged over both the masked and unmasked regions is very weakly dependent on $\theta$, and is very nearly $g_{0}$, the unenhanced value. It is easily seen that the average growth rate is

$$
\bar{g}=g \theta=g_{o} \theta^{0.138}
$$

which is consistent with the weak $\theta$ dependence.

During the actual growth process the masked regions are overgrown, and the ratio of exposed $\mathrm{GaN}$ to dielectric material, i.e., $\theta$, decreases continuously. These calculations show that the growth rate should drop dramatically and continuously as overgrowth proceeds. The growth rate enhancement due to the transport effects should follow Eq. (3) as $\theta$ drops throughout the fill-in process. The growth rate will drop to $g_{0}$ when the gap is completely filled.

This model is confirmed by the series of SEM photos in Figure 2, which shows the overgrowth from 1:32, 1:16, and 1:8 mask patterns from the same growth run. Analysis of the SEMs shows that the $1: 32$ area has overgrown the dielectric mask by $\mathrm{d}=12 \mu \mathrm{m}$. The features from the 1:16 pattern have not begun to touch, and thus have growth less than $d=8 \mu \mathrm{m}$. The $1: 8$ features have touched, but are not yet coalesced into a smooth film, and thus the lateral growth distance is around $d=4 \mu \mathrm{m}$. Finally, GaN from a 1:4 pattern not shown coalesced into a smooth film, indicating that $d>2 \mu \mathrm{m}$. Thus, the extent of lateral growth has dropped precipitously with fill-factor. Note that the gap between the upper and lower set of features is simply due to a gap in the lithographically defined mask pattern.

Equation (3) is the model growth rate as a function of $\theta$ at any point in time. This simple differential equation can be integrated to give the growth front as a function of time

$$
x(t)=\left[(a+1) g_{o}(L / 2)^{a} t+x_{o}^{a+1}\right]^{1 /(a+1)},
$$

where $\mathrm{a}=0.862, L$ is $w+m$ (exposed plus masked distances), and $x_{0}$ is $w / 2$. Differentiating Eq. (5) with respect to time yields $g$ of Eq. (3), where $\theta=2 x / L$. 


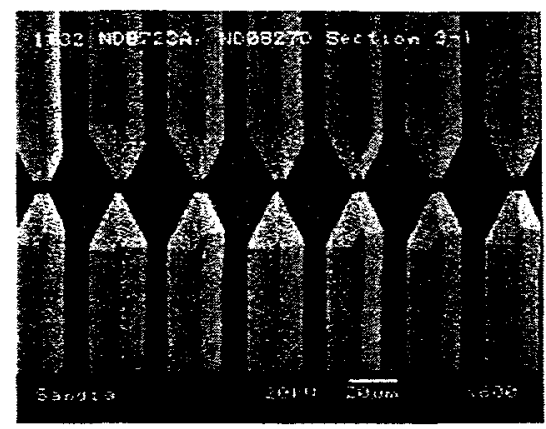

(a)

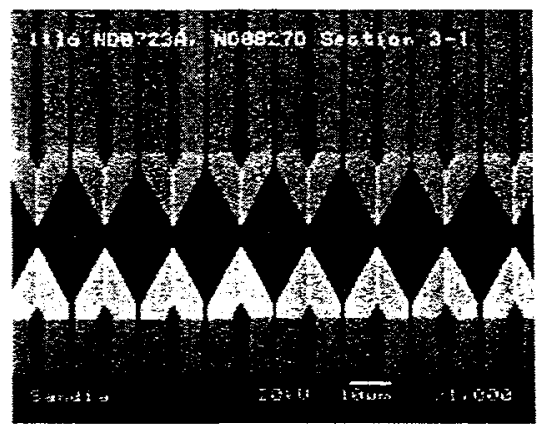

(b)

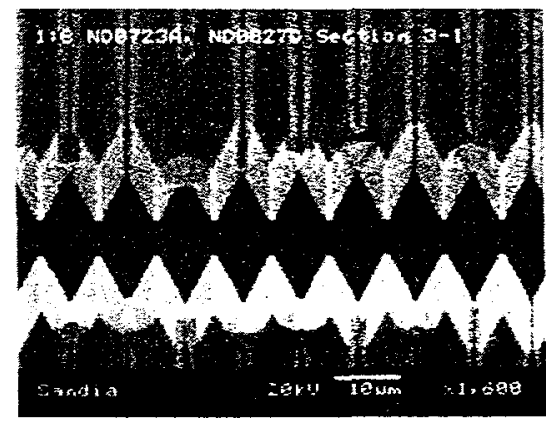

(c)

Figure 2. SEM micrographs showing the extent of lateral overgrowth from patterns of different masked dimensions (a) 1:32 (x600 magnification), (b) 1:16 (x1000), (c) 1:8 (x1600)

Figure 3 compares the lateral growth distance $d=x(t)-x_{0}$ for two sets of mask patterns, with the masked lines running along the $11(-2) 0$ and $1(-1) 00$ directions in Figure $3 a$ and Figure $3 b$, respectively. All of the parameters in Eq. (5) are known except the unenhanced growth rate $g_{0}$, which is determined from the measured lateral growth distance from the 1:32 pattern, i.e., $\theta=$ 0.0303 . Agreement between the model and experiment is quite good.

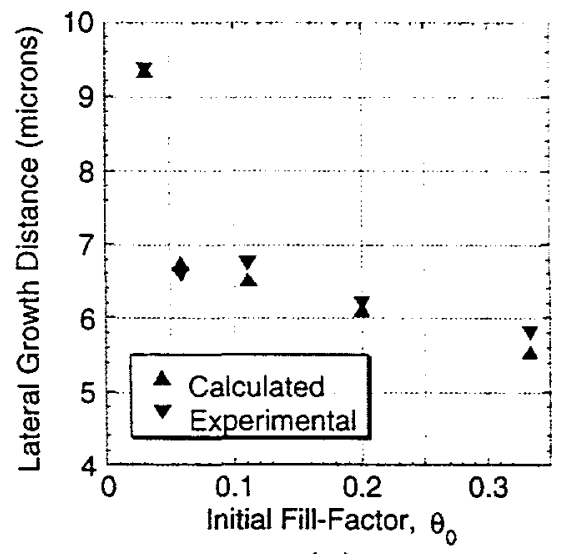

(a)

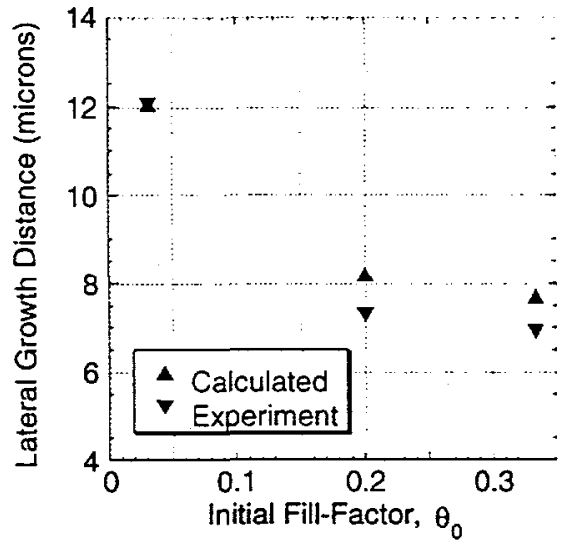

(b)

Figure 3. Comparison of lateral growth distance as a function of initial fill-factor measured from the SEM micrographs and calculated from Eq. (5).

\section{AN ALTERNATIVE COALESCENCE MECHANISM}

SEM "snap-shots" reveal that coalescence can also occur via a mechanism quite different from that observed in previous ELO studies. Typically, coalescence of adjacent features results from each growth front progressing laterally until the region in between is filled completely. [11] However as shown in Figure 4a, the adjacent lateral growth fronts have only just come to the point of convergence and yet the vertical height of the coalescence region has nearly reached the level of the upper-most surfaces. This apparent enhancement in the local growth rate is consistent with the decrease described by the ELO transport model. Since the lateral rate is reduced greatly by the time the convergence point is reached, the creation of a reaction site on the surface more favorable to growth will be conducive to a relatively greater rate. In this case, it is the point of convergence itself, where the lateral growth fronts first come in contact, which serves as the reaction site for initiating new growth. This observation of local growth rate 
enhancement under conditions where lateral growth is limited by TMG gas-phase transport, also supports previous suggestions that competition by various crystal faces for nutrients diffusing on the surface has an important role in GaN growth [14].

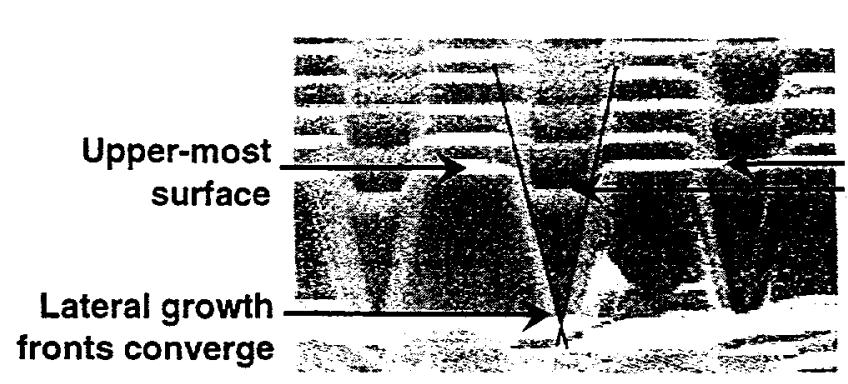

(a)

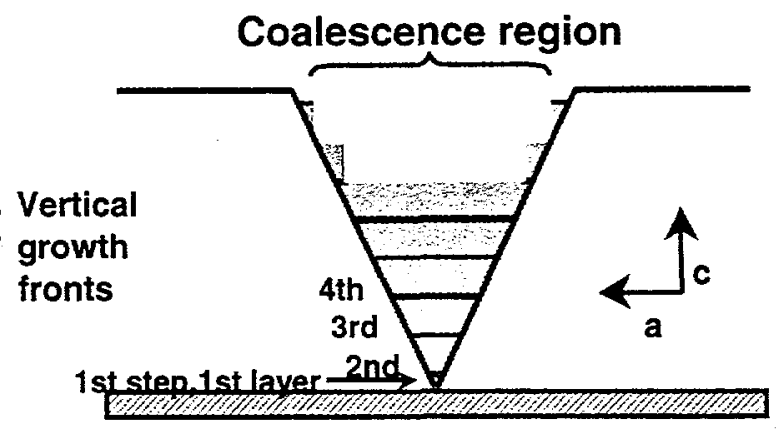

(b)

Figure 4. (a) SEM of a selective area growth dot pattern showing that adjacent lateral growth fronts have only just touched. (b) Diagram of the step-flow coalescence mechanism.

As proposed in Figure 4b, the first "step" and the first layer of GaN is initiated at the moment of contact between adjacent growth fronts. This new surface provides an avenue for rapid growth of one layer at a time since prior to convergence, the lateral progress of a single layer is inhibited by the inert mask. Each new surface then defines new steps along the initial growth fronts for the nucleation of each subsequent layer, as suggested by the slight upward curvature of the vertical growth front end-points in Figure 4a. Thus, a step-flow coalescence mechanism is responsible for the rapid lateral rate. This in turn drives the vertical rate, filling the coalescence region. Since crystal growth in the coalescence region begins at a single point or line, films having more uniform properties may result. The data in the next section support this.

\section{CATHODOLUMUNESCENCE}

Cathodoluminescence (CL) with a sub-micron spatial resolution, is a useful method to characterize the optical quality of $\mathrm{GaN}$ films. The $\mathrm{CL}$ was measured from samples of both planar GaN and smooth coalesced films grown with the ELO technique. Using an SEM to excite luminescence (100pA beam current at $10 \mathrm{keV})$, [15] band-edge or deep-level defect band emission imaging was selected with filters.

Figure 5a shows band-edge emission at $363 \mathrm{~nm}$ for a $144 \mu \mathrm{m}^{2}$ area of non-ELO GaN. The mottled appearance is characteristic of $\mathrm{GaN}$ and has been attributed to threading dislocations in the material that appear as non-radiative recombination centers [16]. Figure 5b shows bandedge emission for a similar area of a fully coalesced ELO region grown using a 1:1 mask pattern on the same wafer. SEM reveals this region to have a smooth surface, and as shown in the figure, the luminescence intensity does not show a periodicity corresponding to the underlying mask. In addition, the luminescence intensity is much more uniform than for the planar GaN, suggesting a reduction of the threading dislocation density due to the ELO growth. The overall luminescence intensity of the ELO sample is approximately $25 \%$ less than the intensity of the non-ELO material. This suggests that other types of non-radiative point defects may be introduced that affect the overall optical quality of the material.

Additional CL tests on samples with varying fill factor show that as the amount of ELO material is increased relative to the nucleation area, the overall intensity of band-edge and deep level emission increased. Also, as the amount of ELO material is increased, the uniformity of the $\mathrm{CL}$ is decreased. This illustrates the important trade-off between luminescence uniformity and luminescence intensity in the development of a $\mathrm{GaN}$ base for the fabrication of devices. 


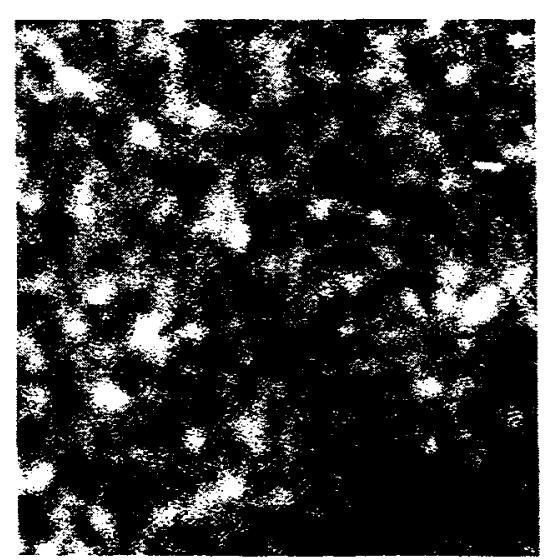

(a)

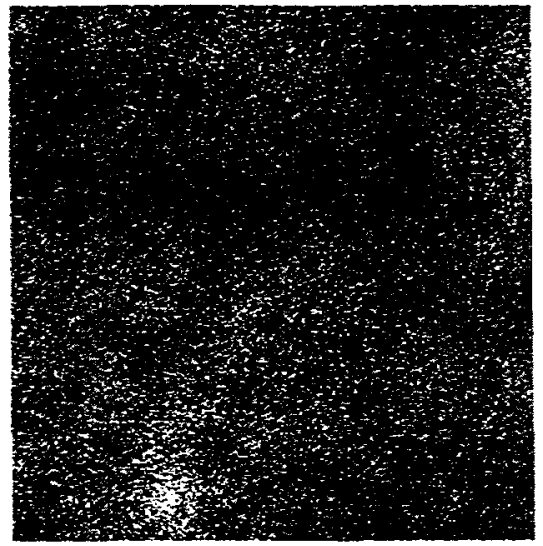

(b)

Figure 5. (a) $\mathrm{CL}$ image at $363 \mathrm{~nm}$ of non-ELO GaN $\left(4 \mu \mathrm{m}\right.$ grown at $\left.1050^{\circ} \mathrm{C}\right)$. (b) $\mathrm{CL}$ image of ELO GaN from a $1: 1$ pattern $\left(4 \mu \mathrm{m}\right.$ grown at $\left.1050^{\circ} \mathrm{C}\right)$.

\section{ACKNOWLEDGMENTS}

This work was performed at Sandia National Laboratories and supported by the U. S. Department of Energy under contract number DE-AC04-94AL85000. Technical support provided by Jeff Figiel, Mike Banas, and R. Guild Copeland is noted with appreciation.

\section{REFERENCES}

1. S. Nakamura, T. Mukai, M. Senoh, Appl. Phys. Lett. 64, 1687-1689 (1994).

2. S. Keller, B.P. Keller, Y.F. Wu, B. Heying, D. Kapolnek, J.S. Speck, U. K. Mishra, and S.P. DenBaars, Appl. Phys. Lett. 68, 15254 (1996).

3. S. Nakamura, M. Senoh, S. Nagahama, N. Iwasa, T. Yamada, T. Matsushita, H. Kiyoku, Y. Sugimoto, T. Kozaki, H. Jumemoto, M. Sano, and K. Chocho, Appl. Phys. Lett, 72, 211 (1998)

4. N. G. Weimann, L.F. Eastman, D. Dharanipal, H.M. Ng, and T.D. Moustakas, J. Appl. Phys. 83, 3656 (1998).

5. D. Kapolnek, S. Keller, R. Vetury, R.D. Underwood, P. Kazodoy, S.P. Denbaars, and U.K. Mishra, Appl. Phys. Lett. 71, 1204-1206 (1997).

6. T.S. Zheleva, O.-H. Nam, M.D. Bremser, and R.F. Davis, Appl. Phys. Lett. 71, 2472 (1997).

7. O.-H. Nam, M.D. Bremser, T.S. Zheleva, and R.F. Davis, Appl. Phys. Lett. 71, 2638 (1997).

8. O.-H. Nam, T.S. Zheleva, M.D. Bremser, and R.F. Davis, J. Elec. Mat. 27, 233-237 (1998)

9. H. Marchand, J.P. Ibbetson, P.T. Fini, S. Keller, S.P. DenBaars, J.S. Speck, and U.K. Mishra, Submitted to J. Crystal Growth, (1998).

10. A. Kimura, C. Sasaoka, A. Sakai, A. Usui, Mat. Res. Symp. Proc. 482, 119-124 (1998)

11. B. Beaumont, M. Vaille, G. Nataf, A. Bouille, J.-C. Guillaume, P. Vennegues, S. Haffouz, and Pierre Gibart, MRS Internet J. of Nitride Semiconductor Res. 3, Art. 20 (1998).

12. M. E. Coltrin and R. J. Kee, Mater. Res. Soc. Symp. Proc., 145, 119 (1989).

13. M. E. Coltrin, R. J. Kee, and F. M. Rupley, Int. J. Chem. Kinet., 23, 1111 (1991).

14. S. Kitamura, K. Hiramatsu, and N. Sawaki, Jpn. J. Appl. Phys., 34, L1184 (1995).

15. C. H. Seager, N. A. Missert, D. R. Tallant, and W. L. Warren, J. Appl. Phys. 83, 1153 (1998).

16. S. J. Rosner, E. C. Carr, M. J. Ludowise, G. Girolami and H. I. Erikson, Appl. Phys. Lett. 70, 420 (1997). 